\title{
Quality of Care Indicators for Head and Neck Cancers: The Experience of the European Project RARECAREnet
}

\begin{abstract}
Annalisa Trama ${ }^{1 *}$, Laura Botta ${ }^{1}$, Roberto Foschi ${ }^{1}$, Otto Visser ${ }^{2}$, Josep Maria Borras ${ }^{3}$, Tina Žagar ${ }^{4}$, Maja Primic-Žakelj ${ }^{4}$, Francesca Bella ${ }^{5}$, Nadya Dimitrova ${ }^{6}$, Gemma Gatta ${ }^{1}$ and Lisa Licitra ${ }^{7,8}$ on behalf of the RARECAREnet High Resolution Working Group

${ }^{1}$ Evaluative Epidemiology Unit, Research Department, Fondazione IRCCS Istituto Nazionale dei Tumori, Milan, Italy, ${ }^{2}$ Netherlands Comprehensive Cancer Organisation (IKNL), Utrecht, Netherlands, ${ }^{3}$ Department of Clinical Sciences, The Bellvitge Biomedical Research Institute (IDIBELL), University of Barcelona, Barcelona, Spain, ${ }^{4}$ Epidemiology and Cancer Registry, Institute of Oncology Ljubljana, Ljubljana, Slovenia, ${ }^{5}$ Integrated Cancer Registry of Catania-Messina-Siracusa-Enna, Azienda Ospedaliero-Universitaria Policlinico-Vittorio Emanuele, Catania, Italy, ${ }^{6}$ National Hospital of Oncology, Bulgarian National Cancer Registry, Sofia, Bulgaria, ${ }^{7}$ Department of Oncology and Hemato-Oncology, University of Milan, Milan, Italy, ${ }^{8}$ Head and Neck Cancer Medical Oncology Unit, Department of Medical Oncology, Fondazione IRCCS Istituto Nazionale dei Tumori, Milan, Italy
\end{abstract}

Background: Monitoring and improving quality of cancer care has become pivotal today. This is especially relevant for head and neck cancers since the disease is complex, it needs multi therapy, patients tend to be older, they tend to have comorbidities and limited social support. However, information on quality of care for head and neck cancers is scarce. In the context of the project "Information Network on Rare Cancers" we aimed to identify indicators of quality of care specific for the head and neck cancers management and to measure the quality of care for head and neck cancers in different EU Member States. Markus Brunner, Medical University of Vienna, Austria Pernille Lassen, Aarhus University Hospital, Denmark

*Correspondence: Annalisa Trama annalisa.trama@istitutotumori.mi.it

Specialty section: This article was submitted to Head and Neck Cancer, a section of the journal Frontiers in Oncology

Received: 24 April 2019 Accepted: 13 August 2019 Published: 28 August 2019

Citation:

Trama A, Botta L, Foschi R, Visser O, Borras JM, Žagar T, Primic-Žakelj M, Bella F, Dimitrova N, Gatta G and Licitra L (2019) Quality of Care Indicators for Head and Neck Cancers: The Experience of the European Project RARECAREnet. Front. Oncol. 9:837. doi: 10.3389/fonc.2019.00837
Methods: We defined indicators of quality of care for head and neck cancers based on a multidisciplinary and expert-based consensus process at a European level. To test the proposed indicators, we performed an observational population-based retrospective study in four countries (Ireland, Italy, Netherlands, and Slovenia) in the years 2009-2011.

Results: The main quality indicators identified are: availability of formalized multidisciplinary team, participation in clinical and translational research; timeliness of care, high quality of surgery and radiotherapy, and of pathological reporting. For head and neck cancers, the quality of care did not reach the optimal standards in most of the countries analyzed. A high proportion of patients was diagnosed at an advanced disease stage, showed delays in starting treatment (especially for radiotherapy), and there was only a very limited use of multi therapy.

Conclusions: According to the achieved consensus, indicators of quality of care for head and neck cancers have to cover the patient journey (i.e., diagnosis and treatment). Our results, showed suboptimal quality of care across countries and call for solutions for ensuring good quality of care for head and neck cancer patients in all EU countries. One possible option might be to refer head and neck cancer patients to specialized centers or to networks including specialized centers.

Keywords: head and neck cancers, population based studies, quality of care, quality indicators, integrated care 


\section{INTRODUCTION}

Due to the complexity of oncological care, it is essential to deliver integrated care with optimal alignment and collaboration of several disciplines (1). This is especially relevant for head and neck cancers (HNCs). HNCs involve different anatomical sites, including larynx, oral cavity, oropharynx, hypopharynx, nasopharynx, nasal cavity, and sinuses. The disease is complex, often needs multi therapy, including surgery, radiation, chemotherapy, and/or targeted therapy. Patients tend to be older, to have comorbidities and less social support (2).

In order to improve high-quality oncological care, reliable quality indicators (QIs) are indispensable. This was first highlighted in the USA by the Institute of Medicine's report, "Crossing the Quality Chasm: A New Health System for the twenty-first Century" (3). In Europe, reviews on QI of cancer care were made available by the European Partnership for Action Against Cancer (http://www.epaac.eu) and by the European CanCer Organisation Essential Requirements for Quality Cancer Care (ERQCC) (https://www.ecco-org.eu/Global/News/LatestNews/2017/02/ECCO-Essential-Requirements-for-Quality-

Cancer-Care). With regards to HNCs, quality assurance (QA) has been extensively addressed for HNC radiotherapy $(\mathrm{RT})(4,5)$ and surgery $(6,7)$ and to a lesser extent for medical oncology $(1,8-11)$.

Against this background, in the framework of the Information network on rare cancers (RARECAREnet) project (www. rarecarenet.eu), we defined QIs to measure quality of oncological care for the HNC patient journey (i.e., diagnosis and treatment).

In this paper, we report on: (1) the QIs of cancer care for HNC identified by RARECAREnet; (2) the results of the study testing the proposed QIs in several EU countries.

\section{MATERIALS AND METHODS}

Quality indicators for HNC were defined through a consensus process following the steps below:

1. Identification of published QIs;

2. Discussion of the proposed QIs with an expert panel involving multidisciplinary experts in a dedicated meeting;

3. Population-based observational study in several European countries to test the proposed QIs;

4. Discussions about the results of the observational assessment with the same expert panel in a second meeting in which a final agreement on QIs was achieved with no dissent. During this second meeting, the expert panel confirmed the QIs originally proposed and added new indicators. For the latter, data were not collected because the observational assessment had ended. We refer to them as QIs agreed by consensus only (Table 1).

The HNC multidisciplinary expert panel included faculty members of the European Society for Medical Oncology (ESMO), authors of the ESMO clinical practice guidelines (CPG), representatives of the European Head and Neck Society (EHNS), of the European Society for Radiotherapy and Oncology (ESTRO) and representatives of the European Society of Surgical
Oncology (ESSO). HNC patients were represented by the Italian association of laryngectomized patients (Associazione Italiana Laringectomizzati), which is a member of the Make Sense Campaign of the EHNS, and by the European Cancer Patient Coalition.

The observational assessment was performed in collaboration with population-based cancer registries (CRs): the national CRs of Ireland, Netherlands, and Slovenia, as well as nine Italian regional CRs. The study included only incident squamous cell carcinoma (SCC) of larynx, oral cavity, oropharynx, and hypopharynx, diagnosed in patients $>15$ years old. In Ireland and Netherlands all incident SCC of larynx, oral cavity, oropharynx, and hypopharynx were included in the study. In Slovenia, the study focused only on larynx however, all incident cases of larynx SCC diagnosed in Slovenia were included. In Italy, due to the lack of a national CR, nine CRs representative of the different incidence rates of HNCs considered were included. These nine CRs included all incident cases of larynx, oral cavity, oropharynx, and hypopharynx SCC diagnosed in the geographical areas covered by the selected nine CRs. Table 2 enlists the number of cases included by country and the years of diagnosis.

We developed a data collection protocol with HNC clinical experts and CRs, we established a help desk to answer questions on the data collection and we centralized at the Istituto Nazionale Tumori (INT) data quality checks and analyses. All data obtained from CRs were fully anonymized prior to being accessed centrally at INT. The Ethics Committee of INT was notified about the RARECAREnet project including this retrospective observational study.

For HNCs, clinical stage was adapted from the ESMO CPG (13) breaking down patient populations into:

- Localized: T1-T2, N0, M0

- Advanced: T3-T4, N0, M0, or N+ with any T

- Metastatic: M+

- Unknown.

The treatment combinations for HNCs were defined as follows:

- Surgery alone; RT alone; chemotherapy alone (if each started within 3 months from diagnosis),

- Concomitant chemo-radiotherapy (CRT) (if chemotherapy and radiotherapy started on the same day, or if chemotherapy started 1 day before the radiotherapy or within max 21 days after the start of radiotherapy),

- Surgery + radiotherapy or surgery + concomitant radio-chemotherapy (concomitant if chemotherapy and radiotherapy started on the same day, or chemotherapy started 1 day before the radiotherapy or within max 21 days after the start of radiotherapy),

- Other combinations of treatments,

- No treatment.

\section{RESULTS}

Table 1 summarizes the RARECAREnet quality of care indicators for HNCs. All QIs are listed i.e., QI agreed by consensus 
TABLE 1 | List of quality indicators for head and neck cancers.

Diagnostic management

1. Percentage of patients with a defined stage at diagnosis

Time to start treatment (12) and treatment adherence to clinical guidelines (13)

2. Time to start treatment (time between definitive pathological diagnosis and beginning of surgery or radiotherapy $<1$ month)

3. Time in starting post-operative radiotherapy or concomitant chemo-radiotherapy (<8 weeks from surgery)

4. Percentage of patients with early stage I and II referred for either surgery or radiotherapy

5. Percentage of patients with locally advanced stage III and IV referred for surgery plus post-operative radiotherapy or post-operative chemo-radiotherapy or concomitant chemo-radiotherapy

Quality of surgery and radiotherapy

6. Percentage of complete tumor resection (histological verification of tumor free margins after surgery)

7. Percentage of re-operation within 30 days from main surgery ${ }^{\star}$

8. Percentage of grade $\geq 3$ late toxicities ( $>3$ months after radiotherapy)*

9. Percentage of patients receiving intensity-modulated radiation therapy vs. $\%$ receiving 3D conformal radiation therapy

10. Percentage of patients receiving the appropriate surgery for its stage (e.g., minimal invasive, reconstructive surgery) ${ }^{\star}$

\section{Quality of pathology reports after surgery}

11. Percentage of pathology reports after surgery with a full set of core data items recorded. According to the Royal College of Pathologists (https://www.rcpath.org/ profession/publications/cancer-datasets.html): site and laterality of the carcinoma, maximum diameter of tumor, maximum depth of invasion, histological type of carcinoma, degree of differentiation (grade), pattern of invasion, margin status, lymph node involvement.

Availability of formalized multidisciplinary decision (with member experts on head and neck cancers)*

Participation in clinical and translational research*

*Indicators agreed by consensus within the expert panel only.

TABLE 2 | Number of patients with head and neck cancers (HNCs) included in the study by country with years of diagnosis.

\begin{tabular}{lccccc}
\hline Cancer & \multicolumn{5}{c}{ Number } \\
\cline { 2 - 6 } & Total & Ireland & Italy* & Netherlands & Slovenia \\
\hline HNCs & 8,655 & 1,323 & 928 & 6,185 & 219 \\
Hypopharynx & 790 & 121 & 54 & 615 & 0 \\
Larynx & 3,168 & 449 & 398 & 2,102 & 219 \\
Oral cavity & 2,976 & 428 & 258 & 2,290 & 0 \\
Oropharynx & 1,722 & 325 & 218 & 1,178 & 0 \\
Years of diagnosis & & $2009-2011$ & $2009-2010$ & $2009-2011$ & $2009-2010$
\end{tabular}

*Italy included nine population based cancer registries: Registro Tumori Integrato (Catania and Messina), Palermo, Ragusa, and Siracusa (Sicily-south of Italy); Modena, Parma, Reggio Emilia, and Romagna (Emilia Romagna-centre of Italy); Friuli Venezia Giulia (Friuli Venezia Giulia-north est of Italy).

and assessed by the observational study and QIs agreed by consensus only. It was agreed that optimum management of HNC patients requires active involvement of experts from a wide variety of fields including at least: a head and neck surgeon, a radiation oncologist, a pathologist, a radiologist and a medical oncologist, high quality of surgery and radiotherapy, timely start of treatment (12), optimal supportive care management, and the ability to manage complex patients with multiple health and social care needs.

The observation study was performed on 1,323, 928 and 6,185 cases of hypopharynx, larynx, oral cavity, and oropharynx SCC in Ireland, Italy, and Netherlands, respectively. In Slovenia only larynx SCC were included in study $(N=219)$ (Table 2).
Tables 3-5 summarize the results of the observational assessment for the QIs included in the study by country.

Regarding staging at diagnosis (Table 3), in Netherlands and Slovenia, almost all patients were staged (unknown stage $<5 \%$ ). In Italy and Ireland, stage was unknown in about one out of six and one out of four patients, respectively. Most of hypopharynx, oropharynx, and oral cavity cancer patients were diagnosed with an advanced disease stage across all countries.

Table 4 reports the results for the timeliness in starting treatment and the adherence to CPG (Indicators 2, 3, 4, and 5). Many HNC patients started treatment with curative intent (surgery or RT) $>1$ month after the diagnosis. In Italy, $60 \%$ of HNC patients started the treatment within 1 month from diagnosis, in all the other countries the proportion starting the treatment on time decreased to $40 \%$. Most of the HNC patients started adjuvant treatment $<8$ weeks after surgery ranging from $52 \%$ in Italy to $79 \%$ in Netherlands. The only exception was Ireland with $33 \%$ of HNC patients starting adjuvant within the recommended number of days. Adherence to CPG was high for HNC patients with localized disease stage (Indicator 4: 72$79 \%$ ) but low for HNC patients with advanced disease stage (Indicator 5: 19-44\%). Differences were observed in the use of surgery and radiotherapy across head and neck sites and countries. In Italy, surgery was the main treatment for all HNCs, in the other countries RT was the main treatment for larynx cancers and surgery for the other sites (data available from the corresponding author).

Table 5 describes the quality of surgery and of the pathological report after surgery (Indicators 6 and 11). CRs did not find adequate information on type of RT thus results are not reported for the indicator 9. The proportion of HNC patients with complete tumor resection after a surgery with curative intent, 
TABLE 3 | Diagnostic management for head and neck cancers illustrated for larynx and other sites of the head and neck (i.e., hypopharynx, oral cavity and oropharynx), by country.

Diagnostic management

\begin{tabular}{|c|c|c|c|c|c|c|c|c|c|c|}
\hline \multirow[t]{2}{*}{ Country } & \multicolumn{10}{|c|}{ Indicator 1. Percentage of patients with a defined stage at diagnosis } \\
\hline & $N$ & $\%$ L & $\% \mathbf{A}$ & $\% \mathbf{M +}$ & $\%$ Missing & $N$ & $\%$ L & $\%$ A & $\% \mathbf{M}+$ & $\%$ Missing \\
\hline Ireland & 449 & 40 & 35 & 3 & 22 & 874 & 17 & 55 & 4 & 24 \\
\hline Italy & 398 & 50 & 30 & 4 & 16 & 530 & 24 & 49 & 8 & 19 \\
\hline Netherlands & 2,102 & 58 & 37 & 1 & 4 & 4,083 & 38 & 55 & 2 & 4 \\
\hline
\end{tabular}

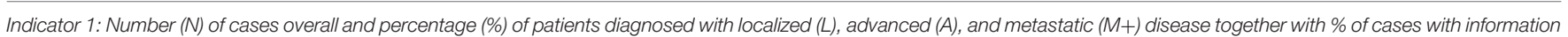
on stage missing, by country.

ranged from $56 \%$ in Ireland to $>70 \%$ in Netherland and Slovenia. The resection margin was unknown in $27 \%$ of cases in Ireland and in $<15 \%$ of cases in the other countries. The pathological report after surgery included all necessary information in a minority of cases (from $1 \%$ in Slovenia to $24 \%$ in Italy). However, in most cases (80-90\%), at least site and laterality of the tumor, histological type and grade were reported. The information less often described were maximum depth of invasion and the pattern of invasion (data available from the corresponding author).

\section{DISCUSSION}

We proposed QIs of cancer care for HNCs based on a multidisciplinary and expert-based consensus process at European level. The proposed QIs cover two critical steps of the patient journey (i.e., diagnosis and treatment) and are easy to collect at the hospital as well as at the population level (i.e., from CRs or administrative data sources). Previous QA for HNCs focused on surgery $(6,7)$ and RT $(4,5)$. In addition, extensive research have supported the important role of multidisciplinary team (MDT) care for HNCs (2, 14-17).

The indicators proposed for surgery include: the presence of a multidisciplinary tumor board advising on more than $90 \%$ of HNCs, the capacity to perform all necessary imaging, the existence of clinical pathways, collaboration with paramedical services, institutional guidelines' hygiene standards being monitored by an institutional board, clinical trial data managers, reports on surgical procedures as indicated by the American Academy of Otolaryngology, Head and Neck Surgery, pathology reports as indicated by the Royal College of Pathologists dataset for histopathology reporting, established reporting system for undesirable events (7).

General radiation oncology (RO)-QIs measure efficiency, waiting time, accuracy of medical records, percentage of cases discussed in a MDT setting, treatment planning based on CT, frequency of verification of treatment portal, measures of physics quality control adequacy, and patient satisfaction (18). For HNC, the National Quality Measures Clearinghouse identified two radiation oncology-related QI i.e., complete follow-up documented for patients receiving RT for glottic cancer and patients receiving post-operative head and neck RT 6 weeks after surgery or longer (4). Guidelines for the delineation of the primary tumor clinical target volumes are also available (19-21). In addition, for HNCs, it is internationally agreed that RT-QA is important, that a radiation oncologist should not practice HNC without adequate training, and that a RT-QA program should be available in RO departments treating HNC. To date, there is no strict international consensus exists on the best model of RT-QA, neither in relation to HNC nor otherwise. However, the benefits of RT-QA and peer review suggest that this has to be incorporated into routine clinical practice. Technology is evolving at a rapid rate: machine learning, artificial intelligence, deformable registration, and radiomics may bring additional refinements to the peer review process. Peer review will form an important component of adaptive treatment, but before this is implemented we will need to consider how to best add this additional burden to head and neck departments (5).

Our QIs include the quality of both, surgery and RT and support the importance of MDT care, of timely start of treatment and of the quality of the pathology reports. A limitation of our QIs is that they do not address quality in systemic therapy. Quality assurance in the medical arena arrived most recently in comparison to other fields. At the beginning of the 90s, the European organization for research and treatment of cancer (EORTC) addressed issues related to the practice of chemotherapy delivery and the quality of data reporting. Furthermore, the EORTC quality assurance committee proposed a minimal set of quality control procedures to be implemented by all EORTC groups (10). However, QA for medical oncology in HNCs was not developed further. A major problem in HNCs medical oncology is the dose intensity in multi therapy. HNC patients with locally advanced disease stage treated with CRT experience moderate/severe side-effects limiting their tolerance to receive the intended cisplatin dose intensity (22). Chemotherapy modifications (dose reductions/delays/omissions) are common (23-40\%) $(23,24)$. The consequence of cumulative cisplatin dose reduction is uncertain although some reports suggest a possible detrimental impact on survival $(22,23,25)$. Discrepancies in treatment adherence expressed as proportion of patients who received all 


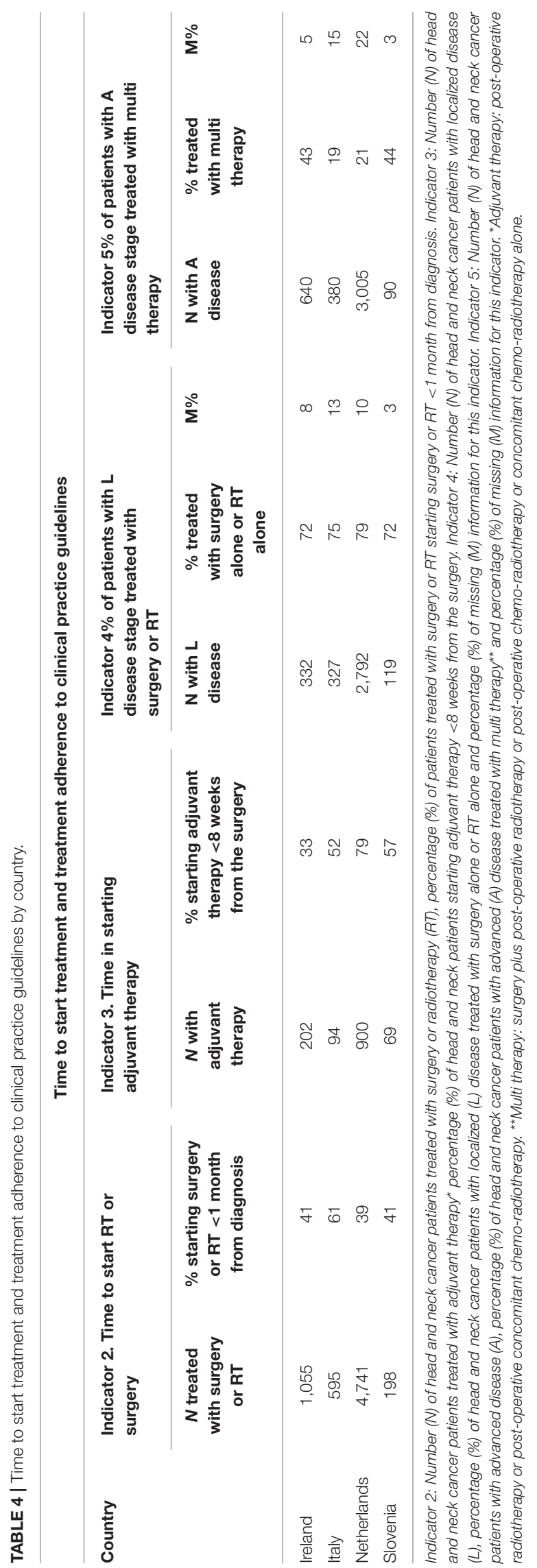

planned cycles of chemotherapy could therefore be used as a QI for systemic therapy in HNC. In this regard, there is an important role for the clinician, not only to stimulate patients to adhere to the treatment schedule, but also to provide optimal supportive care in order to make it tolerable for the patient (22).

Previous studies looking at the quality of integrated care for HNC patients proposed structure and process indicators (e.g., availability of MDT, of an integrated care pathway, of a case manager, of electronic patient information system) $(26,27)$. Other studies proposed an extensive list of outcome, process and structure indicators that needed to be practically tested so to limit the QIs number (1). Although interesting, these studies report about one country-specific context. We tested the QIs in an observational study performed in four different European countries. The data collection, based on clinical dossiers and administrative database, was undertaken with an acceptable proportion of missing $(<20-25 \%)$ in all countries and without major problems in the data collection but for $1 \mathrm{RT}$ indicator. The expert panel agreed that the results of the observational study gave a good description of the quality of care for HNCs in the studies of all the countries, confirming the reliability and validity of the QI proposed in measuring quality of care.

We found that quality of care of HNC does not reach optimal standards in some of the countries analyzed. Many patients were diagnosed an advanced disease stage, which is associated with a worse prognosis (28). Another major problem was treatment delay. This happened most likely when patients were treated with RT, which could impact on their prognosis (29). Possible explanations include possible delays in referral to $\mathrm{RO}$ and concentration of radiotherapy facilities in a few centers with limited resources. A recent survey on radiotherapy capacities in Europe showed significant variability among countries and a lack of RT infrastructures (30). The adherence to CPG for treatment was good. However, we observed a very limited use of multi therapy for advanced-stage patients. This is relevant considering that they are associated with a higher survival (31). We observed inadequate surgery and poor quality of the pathological reports after surgery, which is a matter of concern considering that pathological reports support treatment decisions after surgery.

Our study adds evidence to previous national studies in which compliance of HNCs care to hospital or national CPG was considered a quality care marker. A Dutch study reported a compliance rate of $91 \%$ (32), one study from the USA $86 \%$ (33), going down to $57 \%$ in patients with persistent or recurrent HNCs who were referred to an expert centre (34).

Limitations of our study include the retrospective design and the potential errors in coding associated with the kind of sources of clinical data and their inherent variability in quality of reporting, although every effort was made to standardize data collection and reduce missing data. Strengths are the centralization of data quality checks and analyses along with the population-based nature of this effort, which is essential to generate real-world data.

Our results showed suboptimal standards of quality of care in some of the countries analyzed and call for solutions to increase quality care for HNC patients to ensure high quality of care 
TABLE 5 | Quality of surgery and of the pathological report after surgery.

\begin{tabular}{|c|c|c|c|c|c|c|}
\hline \multicolumn{7}{|c|}{ Quality of surgery and of the pathological report after surgery } \\
\hline \multirow[t]{2}{*}{ Country } & \multicolumn{3}{|c|}{$\begin{array}{l}\text { Indicator } 6 \% \text { of complete tumor } \\
\text { resection }\end{array}$} & \multicolumn{3}{|c|}{$\begin{array}{l}\text { Indicator } 11 \% \text { of pathology reports } \\
\text { with all core data }\end{array}$} \\
\hline & $\begin{array}{l}N \text { treated with surgery } \\
\text { with curative intent }\end{array}$ & $\%$ of R0 & $\mathbf{M} \%$ & $\begin{array}{l}N \text { surgically } \\
\text { treated }\end{array}$ & $\begin{array}{c}\% \text { of post-surgery } \\
\text { pathological report with } \\
\text { all core data }\end{array}$ & $\mathbf{M} \%$ \\
\hline Ireland & 602 & 56 & 27 & NA & NA & \\
\hline Italy & 516 & 62 & 13 & 474 & 24 & 2 \\
\hline Netherlands & 2,728 & 74 & 13 & $128^{\star \star}$ & $16^{\star \star}$ & 0 \\
\hline Slovenia & 88 & 75 & 9 & 87 & 1 & 29 \\
\hline
\end{tabular}

across EU countries. One way of increasing quality of care for HNC patients is to refer them to specialized centers or networks involving specialized centers. Thus, the available evidence show that in the high volume context quality of care is ensured in the entire patient journey:

1) The specialized MDT, which takes considerable time, effort and financial resources works better (35); furthermore the presence of a MDT in high-volume and referral cancer centers is associated with better therapeutic decision (35);

2) The minimal level of quality of surgery is most likely to be reached considering the structural and process criteria identified by the QA programme and the number of major head and neck procedures that should be performed by a leading surgeon per year (7),

3) Experienced clinicians are available to deliver complex HNC RT treatment most accurately and to peer review RT complex plans ensuring RT-QA program (5).

Furthermore, high volume seems to be associated also with better outcomes for HNCs (36-38).

Centralization for many rare cancers including HNCs is still limited in many countries in Europe (39). Gatta et al. reported that for HNCs, $75 \%$ of patients were centralized in two top hospitals in Slovenia ( 2 million population, 266 treatments per hospital per year), and 12 top hospitals in the Netherlands (17 million population, 201 treatments per hospital per year). The level of centralization was lower in the other countries included in the study, resulting in a caseload of 145 treatments/year on overage in each of the 10 Bulgarian top hospitals, 106 treatments/year on average in each of the 29 top Belgian hospitals, 83 treatments/year on average in each of the top six hospitals in Finland, 77 treatments/year on average in the two top hospitals in Navarra, and 63 treatments/year on average in the top seven hospitals in Ireland (39).

It follows that the centralization of care, although hardly feasible for all HNC patients, should be an objective to be pursued. We strongly believe that this objective can only be achieved by progressively making it a national health care policy priority.

The quality ensured by the case volume could be explained by several factors: organization, facilities, processes of care, quality assurance programs, professional expertise, adherence to clinical protocols, technology. In this context, it will be important to detect which of these factors influences final outcomes among HNC patients. We are currently performing additional analyses to assess the relationship between hospital volume and the QIs proposed. We are also trying to assess whether the proposed QIs can explain the observed higher survival observed in high volume centers. In this paper, we present the QI and the process behind their definition. Furthermore, we provide data on quality of care for HNC patients across four countries. This study may be a starting point showing the variability in clinical practice as well as the need to make every effort to increase quality of care for HNC patients in all EU countries.

\section{DATA AVAILABILITY}

All datasets generated for this study are included in the manuscript and/or the supplementary files.

\section{ETHICS STATEMENT}

The study was approved by the Ethics Committee of the IRCCS National Cancer Institute. Being a retrospective study on a rare and lethal disease, the written consent was not necessary.

\section{AUTHOR CONTRIBUTIONS}

AT: study design, supervision of data collection, results interpretation, and manuscript writing. $\mathrm{LB}$ and RF: study design and statistical analyses. OV: study design, data collection, results interpretation, and manuscript revision. JB: manuscript revision. TŽ, MP- $\check{Z}$, and FB: data collection, results 
interpretation, and manuscript revision. ND, GG, and LL: study design, results interpretation, and manuscript revision.

\section{FUNDING}

This work was supported by the European Commission through the Consumers, Health, Agriculture, and Food Executive Agency (Chafea) [grant number 2000111201]lnformation network on rare cancers-RARECARENet. The funders had no role in study design, data collection and analysis, decision to publish, or preparation of the manuscript.

\section{ACKNOWLEDGMENTS}

We thank the expert panel for contributing to the definition of quality indicators for head and neck cancers: European Head and Neck Society-Rene Leemans; European Society for Medical Oncology-Lisa Licitra; European Society for Radiotherapy and Oncology-Marco Krengli, Daniel Zips; European Society of Surgical Oncology-Sergio Sandrucci, Associazione Italiana Laringectomizzati-Umberto Tassini; European Cancer Patient Coalition-Kalliopi Anna Christoforidi, Francesco De Lorenzo.

\section{REFERENCES}

1. van Overveld LF, Braspenning JC, Hermens RP. Quality indicators of integrated care for patients with head and neck cancer. Clin Otolaryngol. (2017) 42:322-9. doi: 10.1111/coa.12724

2. Licitra L, Keilholz U, Tahara M, Lin JC, Chomette P, Ceruse P, et al. Evaluation of the benefit and use of multidisciplinary teams in the treatment of head and neck cancer. Oral Oncol. (2016) 59:73-9. doi: 10.1016/j.oraloncology.2016.06.002

3. Institute of Medicine. Crossing the Quality Chasm: A New Health System for the Twenty-first Century. Washington, DC: National Academy Press (2001).

4. Albert JM, Das P. Quality indicators in radiation oncology. Int J Radiat Oncol Biol Phys. (2013) 85:904-11. doi: 10.1016/j.ijrobp.2012.08.038

5. McDowell L, Corry J. Radiation therapy quality assurance in head and neck radiotherapy-moving forward. Oral Oncol. (2019) 88:180-5. doi: 10.1016/j.oraloncology.2018.11.014

6. Andry G, Hamoir M, Leemans CR. Quality assurance in head and neck surgery: special considerations to catch up. Eur Arch Otorhinolaryngol. (2018) 275:2145-9. doi: 10.1007/s00405-018-5046-9

7. Simon C, Dietz A, Leemans CR. Quality assurance in head and neck cancer surgery: where are we, and where are we going? Curr Opin Otolaryngol Head Neck Surg. (2019) 27:151-6. doi: 10.1097/MOO.0000000000000519

8. Collins R, Flynn A, Melville A, Richardson R, Eastwood A. Effective health care: management of head and neck Cancers. Qual Saf Health Care. (2005) 14:144-8. doi: 10.1136/qshc.2005.013961

9. National Institute for Clinical Excellence (NICE). Guidance on Cancer Services: Improving Outcomes in Head and Neck Cancers: The Manual. London: NICE (2004).

10. Therasse P, De Mulder PH. Quality assurance in medical oncology within the EORTC. European Organisation for Research and Treatment of Cancer. Eur J Cancer. (2002) 38:S152-4. doi: 10.1016/S0959-8049(01)00437-3

11. Digue L, Pedeboscq S. Quality assurance in head and neck medical oncology. Bull Cancer. (2014) 101:486-95. doi: 10.1684/bdc.2014.1968

12. Jensen AR, Nellemann HM, Overgaard J. Tumor progression in waiting time for radiotherapy in head and neck cancer. Radiother Oncol. (2007) 84:5-10. doi: 10.1016/j.radonc.2007.04.001

\section{THE RARECARENET HIGH RESOLUTION WORKING GROUP}

Belgian Cancer Registry: Elizabeth Van Eycken, Kris Henau; Bulgaria National Cancer Registry: Nadia Dimitrova; Finland National Cancer Registry: Maarit Leinonen; Ireland National Cancer Registry: Harry Comber, Fiona F. Dwane; Italy: Registro tumori del Friuli Venezia Giulia: Silvia Birri, Diego Serraino; Registro tumori della provincia di Modena: Claudia Cirilli, Martina Manni; Registro tumori della provincia di Parma: Maria Michiara, Anita Rimanti; Registro tumori Reggiano: Lucia Mangone, Massimo Vicentini; Registro tumori della Romagna: Fabio Falcini, Stefania Giorgetti; Registro tumori Integrato (RTI) di CT-ME-SR-EN: Salvatore Sciacca, Francesca Bella; Registro tumori di Palermo: Rosanna Cusimano, Rosalba Amodio; Registro tumori ASP Ragusa per le provincie di Ragusa e Caltanissetta: Rosario Tumino, Patrizia Concetta Rollo; Registro tumori della provincia di Siracusa: Anselmo Madeddu, Antonino Ziino Colanino; Registro tumori di Trapani: Giuseppa Candela, Tiziana Scuderi; Slovenia National Cancer Registry: Maja Primic-Žakelj, Tina Žagar; The Netherlands Otto Visser, Jan Maarten van der Zwan; Fondazione IRCCS Istituto Nazionale dei Tumori, Milano: Nadia Hindi, Martina Imbimbo, Riccardo Capocaccia.

13. Grégoire V, Lefebvre JL, Licitra L, Felip E, EHNS-ESMO ESTRO Guidelines Working Group. Squamous cell carcinoma of the head and neck: EHNS-ESMO-ESTRO Clinical Practice Guidelines for diagnosis, treatment and follow-up. Ann Oncol. (2010) 21:vi184-6. doi: $10.1093 /$ annonc/mdq185

14. De Felice F, Tombolini V, de Vincentiis M, Magliulo G, Greco A, Valentini V, et al. Multidisciplinary team in head and neck cancer: a management model. Med Oncol. (2018) 36:2. doi: 10.1007/s12032-018$1227-\mathrm{z}$

15. Badran KW, Heineman TE, Kuan EC, St. John MA. Is multidisciplinary team care for head and neck cancer worth it? Laryngoscope. (2018) 128:1257-8. doi: 10.1002/lary.26919

16. Bergamini C, Locati L, Bossi P, Granata R, Alfieri S, Resteghini C, et al. Does a multidisciplinary team approach in a tertiary referral centre impact on the initial management of head and neck cancer? Oral Oncol. (2016) 54:54-7. doi: 10.1016/j.oraloncology.2016.01.001

17. Brunner M, Gore SM, Read RL, Alexander A, Mehta A, Elliot M, et al. Head and neck multidisciplinary team meetings: effect on patient management. Head Neck. (2015) 37:1046-50. doi: 10.1002/hed.23709

18. Cionini L, Gardani G, Gabriele P, Magri S, Morosini PL, Rosi A, et al. Quality indicators in radiotherapy. Radiother Oncol. (2007) 82:191-200. doi: 10.1016/j.radonc.2006.12.009

19. Lee AW, Ng WT, Pan JJ, Poh SS, Ahn YC, AlHussain H, et al. International guideline for the delineation of the clinical target volumes (CTV) for nasopharyngeal carcinoma. Radiother Oncol. (2018) 126:25-36. doi: 10.1016/j.radonc.2017.10.032

20. Grégoire V, Evans M, Le QT, Bourhis J, Budach V, Chen A, et al. Delineation of the primary tumour Clinical Target Volumes (CTV-P) in laryngeal, hypopharyngeal, oropharyngeal and oral cavity squamous cell carcinoma: AIRO, CACA, DAHANCA, EORTC, GEORCC, GORTEC, HKNPCSG, HNCIG, IAG-KHT, LPRHHT, NCIC CTG, NCRI, NRG Oncology, PHNS, SBRT, SOMERA, SRO, SSHNO, TROG consensus guidelines. Radiother Oncol. (2018) 126:3-24. doi: 10.1016/j.radonc.2017.10.016

21. Grégoire V, Ang K, Budach W, Grau C, Hamoir M, Langendijk JA, et al. Delineation of the neck node levels for head and neck tumors: a 2013 update. DAHANCA, EORTC, HKNPCSG, NCIC CTG, NCRI, 
RTOG, TROG consensus guidelines. Radiother Oncol. (2014) 110:172-81. doi: 10.1016/j.radonc.2013.10.010

22. Szturz P, Wouters K, Kiyota N,Tahara M, Prabhash K, Noronha $\mathrm{V}$, et al. Low-dose vs. high-dose cisplatin: lessons learned from 59 chemoradiotherapy trials in head and neck cancer. Front Oncol. (2019) 9:86. doi: 10.3389/fonc.2019.00086

23. Nguyen-Tan PF, Zhang Q, Ang KK, Weber RS, Rosenthal DI, Soulieres D, et al. Randomized phase III trial to test accelerated versus standard fractionation in combination with concurrent cisplatin for head and neck carcinomas in the Radiation Therapy Oncology Group 0129 trial: long-term report of efficacy and toxicity. J Clin Oncol. (2014) 32:3858-66. doi: 10.1200/JCO.2014.55.3925

24. O'Sullivan B, Huang SH, Siu LL, Waldron J, Zhao H, Perez-Ordonez B, et al. Deintensification candidate subgroups in human papillomavirus-related oropharyngeal cancer according to minimal risk of distant metastasis. J Clin Oncol. (2013) 31:543-50. doi: 10.1200/JCO.2012.44.0164

25. Strojan P, Vermorken JB, Beitler JJ, Saba NF, Haigentz M Jr., Bossi P, et al. Cumulative cisplatin dose in concurrent chemoradiotherapy for head and neck cancer: a systematic review. Head Neck. (2016) 38(Suppl 1):E2151-8. doi: $10.1002 /$ hed.24026

26. Ouwens MM, Marres HA, Hermens RR, Hulscher MM, van den Hoogen FJ, Grol RP, et al. Quality of integrated care for patients with head and neck cancer: development and measurement of clinical indicators. Head Neck. (2007) 29:378-86. doi: 10.1002/hed.20532

27. Ouwens MM, Hermens RR, Hulscher MM, Merkx MA, van den Hoogen FJ, Grol RP, et al. Impact of an integrated care program for patients with head and neck cancer on the quality of care. Head Neck. (2009) 31:902-10. doi: 10.1002/hed.21041

28. Gatta G, Botta L, Sánchez MJ, Anderson LA, Pierannunzio D, Licitra L, et al. Prognoses and improvement for head and neck cancers diagnosed in Europe in early 2000s: The EUROCARE-5 population-based study. Eur. J. Cancer. (2015) 51:2130-43. doi: 10.1016/j.ejca.2015.07.043

29. Mackillop WJ. Killing time: the consequences of delays in radiotherapy. Radiother Oncol. (2007) 84:1-4. doi: 10.1016/j.radonc.2007.05.006

30. Grau C, Defourny N, Malicki J, Dunscombe P, Borras JM, Coffey M, et al. Radiotherapy equipment and departments in the European countries: final results from the ESTRO-HERO survey. Radiother Oncol. (2014) 112:155-64. doi: 10.1016/j.radonc.2014.08.029

31. Rudat V, Wannenmacher M. Role of multimodal treatment in oropharynx, larynx, and hypopharynx cancer. Semin Surg Oncol. (2001) 20:66-74. doi: $10.1002 /$ ssu. 1018
32. Hamaker ME, Smorenburgc CH, Bund RJ, de Kuyper GT, van Munster BC, de Rooij SE, et al. Age-related differences in guideline adherence for head and neck cancer. J Geriatric Oncol. (2012) 3:329-36. doi: 10.1016/j.jgo.2012.07.004

33. Lewis CM, Nurgalieva Z, Sturgis EM, Lai SY, Weber RS. Improving patient outcomes through multidisciplinary treatment planning conference. Head Neck. (2016) 38:E1820-5. doi: 10.1002/hed.24325

34. Lewis CM, Hessel AC, Roberts DB, Guo YZ, Holsinger FC, Ginsberg LE, et al. Prereferral head and neck cancer treatment: compliance with National Comprehensive Cancer Network Treatment Guidelines. Arch Otolaryngol Head Neck Surg. (2010) 136:1205-11. doi: 10.1001/archoto. 2010.206

35. Alfieri S, Orlandi E, Bossi P. The case volume issue in head and neck oncology. Curr Treat Options Oncol. (2017) 2718:65. doi: 10.1007/s11864-0170507-8

36. Nieman CL, Stewart CM, Eisele DW, Pronovost PJ, Gourin CG. Frailty, hospital volume, and failure to rescue after head and neck cancer surgery. Laryngoscope. (2018) 128:1365-70. doi: 10.1002/lary.26952

37. Eskander A, Merdad M, Irish JC, Hall SF, Groome PA, Freeman JL, et al. Volume-outcome associations in head and neck cancer treatment: a systematic review and meta-analysis. Head Neck. (2014) 36:1820-34. doi: 10.1002/hed.23498

38. Orlandi E, Alfieri S, Simon C, Trama A, Licitra L, RARECAREnet Working Group. Treatment challenges in and outside a network setting: head and neck cancers. Eur J Surg Oncol. (2019) 45:40-5. doi: 10.1016/j.ejso.2018.02.007

39. Gatta G, Capocaccia R, Botta L, Mallone S, De Angelis R, Ardanaz E, et al. Burden and centralised treatment in Europe of rare tumours: results of RARECAREnet a population-based study. Lancet Oncol. (2017) 18:1022-39. doi: 10.1016/S1470-2045(17)30445-X

Conflict of Interest Statement: The authors declare that the research was conducted in the absence of any commercial or financial relationships that could be construed as a potential conflict of interest.

Copyright (c) 2019 Trama, Botta, Foschi, Visser, Borras, Žagar, Primic-Žakelj, Bella, Dimitrova, Gatta and Licitra. This is an open-access article distributed under the terms of the Creative Commons Attribution License (CC BY). The use, distribution or reproduction in other forums is permitted, provided the original author(s) and the copyright owner(s) are credited and that the original publication in this journal is cited, in accordance with accepted academic practice. No use, distribution or reproduction is permitted which does not comply with these terms. 